\title{
Research on the Application of STC Operator Method in TRIZ Innovation Theory
}

\author{
Li Yuyan, Guo Kai, Zhang Xiangmin* \\ Henan University of Science and Technology, 471023 \\ Luoyang, China \\ emaill:5135758@qq.com
}

\begin{abstract}
Smart phones in the design level of innovative space is getting smaller and smaller, and TRIZ innovation theory can quickly solve the problem of invention and technical innovation. This paper using the STC operator method, from the three dimensions of the smart phone size, standby time and cost, do the divergent thinking of the six dimensions, the use of extreme thinking to explore the future development trend of mobile phones. The future trend of smart phones will have intelligent monitoring of human health, remote control smart appliances, easy to carry, long standby time and other aspects.
\end{abstract}

Keywords-TRIZ; Technical innovation; STC; Mobile phone

\section{INTRODUCTION}

TRIZ innovation theory is a theory and method to solve the problem of creativity. It establishes a series of universal tools, a model to solve the problem of innovation and a method of analyzing problems. It pointed out the exploration direction to solve the problem of innovation. Through the analysis of the patent problem research, the theory of TRIZ innovation form the methodology used to solve the problem of invention. This method has not only made great achievements in the field of engineering and technology, but also extended to the fields of natural science, social science, biological science and other fields [1]. It provides scientific methods, principles and tools for people to solve innovative problems. TRIZ innovation theory has been successfully applied to Boeing, SONY, ZTE, Samsung Electronics and other world-renowned high-tech enterprises [2]. Practice has proved that the TRIZ innovation theory has more advantages and advanced than other innovative methods. The theory fully reveals the inherent rules and principles of innovation, at the same time it makes innovation simpler. There are mainly five kinds of innovative thinking methods based on TRIZ theory, namely multi-screen method, goldfish method, STC operator method, RTC operator method and little person method [3]. These methods are based on the objective laws, and guide people to divergent thinking.

In the eighth decade of twenty century, the first generation of mobile phones was developed by Dr. Cooper of MOTOROLA, at that time has been nicknamed the "bricks" or black diamond. With the development of the times, innovation will inevitably become a new engine for the development of science and technology. The phone has changed greatly both in appearance and function. At present, the intelligent mobile phone has developed into a set of video calls, surf the Internet, navigation, photography, video, games and listen to music and other functions. It has variety of styles and price range, at the same time it meets the needs of all ages and class groups [4]. With the advent of the Internet era, the mobile phone has evolved from a communication tool to an intelligent terminal. Smart phones will be less and less space in the innovation of the industrial design level. The rising development way of mobile devices is not in conformity with the trend of "cloud" [5]. Using TRIZ innovation theory, not only follow the objective law, but also predict the future development trend and develop the innovative products with competitive advantage.

\section{THEORY SUMMARY}

\section{A. Overview of TRIZ innovation theory}

1946, the former Soviet scientist Genrich S. Altshuler and his team put forward the theory of inventive problem solving for the first time, which is called TRIZ innovation theory. Through the study of 2.5 million high-level patents, the paper summarizes the development rules of various technologies and the innovative principles and rules to solve various technical contradictions, and puts forward the basic method to solve the problem of invention [6]. This method has not only made great achievements in the field of engineering and technology, but also extended to the fields of natural science, social science, biological science and other fields.

The core idea of TRIZ innovation theory: whether it is simple or complex product system, its core technology all follow the objective law of development, which has the objective law of development and evolution model; with various technical problems, conflicts and contradictions appear constantly and solving, continuously promote the development of evolution; the ideal state of technology system for the development of the resources is to use less resources realize more function [7].

The TRIZ innovation theory mainly includes three parts: the principle of conflict resolution; the standard solution of the field model analysis; the inventive problem solving algorithm. The core of TRIZ innovation theory is the theory of technology evolution. The technical system is in the process of continuous evolution, and the solution of conflict provides impetus for it. The speed of technology system evolution is gradually reduced with the solution of the conflict, and the only way to change the quality of the system is to solve the deep-seated conflicts in its evolution. 
TRIZ innovation theory based on the theory and methodology of the tool system. It has attracted a large number of countries and enterprises, at the same time encourage them to continue to expand the research and practice, and constantly enrich the TRIZ innovation theory and form a series of method system. This theory promoted enterprise innovation ability and made significant economic benefits, at the same time it has made outstanding contributions [8]. The advantages of TRIZ innovation theory is to enable people to quickly and accurately find and analyze the key issues, improve the efficiency of invention; expansion different angles of thinking, provide a rich knowledge base; the invention process logic is stronger and more scientific technology development trend be more reasonable prediction and the inventor be more strategic [9].

There are many innovative methods to solve the problem. The innovation of the traditional approach requires users to have superb skills, rich experience, more knowledge accumulation, but it has a great chance, and the innovation efficiency is generally not high. Based on the theory of TRIZ, the innovative thinking method is feasible and practical, and it can improve the efficiency of solving the problem of innovation. STC operator method to follow the objective laws of multi-dimensional thinking, faster than traditional methods of innovation.

\section{B. Overview of STC operator method}

STC operator method is one of the most innovative thinking methods in TRIZ theory. This method respectively from the size of objects, time and cost of three different aspects of thinking (as shown in Figure 1), breaking the understanding of objects inherent size, time and cost. This is a kind of regular, multi-dimensional divergent thinking, more conducive to the development of new products.

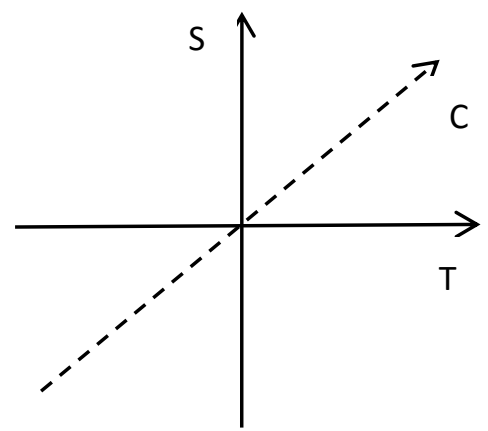

Fig. 1. Size - time - cost coordinates

\section{Rules of STC operator method:}

- Define the size, time and cost of the object.

- Imagine when the size of objects tend to 0 , observe the object changes, design the possible change trend of objects; and then imagine when the object size tends to infinity, observe the object changes, design the possible change trend of objects.

- Imagine when time objects tend to 0 , observe the object changes, design the possible change trend of objects; and then imagine when time tends to infinity when objects, observe the object changes, design the possible change trend of objects.
- Imagine when the object cost tends to 0 , observe the object changes, design the possible change trend of objects; and then imagine when the object cost tends to infinity, observe the object changes, design the possible change trend of objects

In accordance with the above rules to the object, it is convenient for people to observe and study objects from different angles, which is conducive to breaking the shackles of inertial thinking and find solutions to new problems or create new products.

\section{Application analysis of STC operator method in mobile phone innovation}

The STC operator method is a very simple innovation tool. It is one of the objects themselves with different characteristics (size, time, cost) consider separately using the limit way of thinking, through the three aspects of object size, time and cost are extreme assumptions, forecast object change trend, single factors tend to consider produce unexpected ideas, thus creating new objects in line with follow the development trend.

\section{1) Application background}

At present, the mobile phone market has entered the buyer's market led by consumer. Different consumer groups, mobile phone psychological needs are different, can be summarized as the following five kinds: practical needs, aesthetic needs, fashion needs, quality needs and individual needs [10]. In order to meet the different needs of different consumer groups, out of the plight of growth in mobile phone sales, mobile phone industry continues to develop innovation.

\section{2) Problem description}

In order to adapt to the development of the times, the innovation of mobile phone is urgently needed. Mobile innovation can not be limited to appearance, function, cost, etc. How to create a mobile phone conform to the development trend? We can consider the use of STC operator method to explore the mobile phone innovation.

\section{3) Problem analysis}

Currently, the mobile phone screen size from 1.8 inches to 7 inches. The longest standby time is Motorola's Droid Turbo, its standby time reached 10 hours and 42 minutes, called the strongest standby king smart phone. The price of mobile phone ranges from several hundred dollars to ten million dollars. The largest number of mobile phones sold thousands of dollars.

We apply the STC operator method in the innovation process of mobile phone. In the coordinate system of STC operator method, 6 dimensions of divergent thinking are explored in the following 3 dimensions: size, standby time and cost.

a) Suppose the phone size tends to O. In this case, the phone no longer need to operate by hand, liberating people's hands, make life more convenient. How to minimize the size of the phone? Only the chip size of the human body is easy to implant under human skin. We can use the body chip technology, the chip implanted in the body under the skin. This chip can record of personal information, can also record the human health, in the index exceeded the normal value, to prompt or close relatives to transmit information. This technology can also be used for remote control of furniture and appliances. At the same 
time, we can replace the original screen with the retina display. By using the principle of human visual persistence, let the laser in the horizontal and vertical two directions in the specified order of circular scanning, a small area to produce light striking the retina, people will see the image. At present, people have developed a "paper mobile phone", the mobile phone thickness comparable to credit cards, weighs less than iPhone6 weight $1 / 5$, display only $9.4 \mathrm{~cm}$ long.

b) If the mobile phone size tends to infinity. Currently, the largest mobile phone on the market is 7 inches, pixels appear unclear, short standby time, inconvenient to carry a series of problems. If the phone size continues to increase, people will not be able to watch and fast operation of the phone, the phone's innovation does not bring any benefits.

c) Assume that the standby time of mobile phone tends to 0 . With the increase of mobile phone application software, mobile phone standby time is gradually shortened, which brings great inconvenience to daily use. Mobile phone standby time is too short, people can not use the phone, and the phone's innovation does not bring any benefits.

d) If the mobile phone standby time tends to infinity. In the life of the smart phone rankings, ranked first in Motorola's Droid Turbo, its standby time reached 10 hours and 42 minutes. Extend the standby time, so that people can reduce the number of mobile phone charging, reducing the phone suddenly no electricity inconvenience. We can create a mobile phone can absorb solar energy conversion of shell, the phone in the case of light can continue to charge. Mobile phone always maintain a state of electricity, to solve the problem caused by the phone without electricity.

e) Assume that the cost of mobile phones tends to 0 . Currently, there are many low-cost mobile phones on the market, the general quality is poor, weak signal, short standby time, there are security risks for people's lives caused great inconvenience. Mobile phone cost is too low, the mobile phone innovation does not bring any benefits.

f) If the mobile phone cost tends to infinity. In this case, the mobile phone is not only a means of communication, but also become a symbol of identity. At present, there are many companies launched luxury mobile phone models, the majority of the rich love. For example: the British designer for a billionaire from Hong Kong to create a $\$ 15$ million 300 thousand iPhone5. The mobile phone create by a 26 karat diamond, 600 white diamonds, sapphire glass screen and weighs 135 grams 24 carat gold. It is by far the most expensive mobile phone.

\section{CONCLUSION}

This paper using the STC operator method, from 3 aspects of the smart phone, the 6 dimensions of divergent thinking. The future of smart phone will not only have video calls, Internet access, navigation, photography, video, games and listen to music, but also have intelligent monitoring of human health, remote control smart appliances, easy to carry, long standby time and other functions.

\section{RESEARCH LIMITATIONS AND PROSPECTS}

By using the STC operator method of TRIZ innovation theory in the analysis of practical problems in the process of innovation, we found that TRIZ innovation theory based on follow the objective law, make innovation problem easier to solve. Although the STC operator method can not directly solve the problem of innovation, it points out the way to solve the problem of innovation, breaking the inertia thinking and expanding the thinking. The coordinate system used by the STC operator method can also change the factors represented by each coordinate axis according to the actual situation. There is a huge space for development in this area. In this paper, the STC operator method is applied to the innovation process of the mobile phone, which has not changed the representative factors of each coordinate system, and hope to make some contribution to the future development of the mobile phone industry.

\section{ACKNOWLEDGMENTS}

In this paper, the research was sponsored by Henan Provincial Department of Education Science and technology research key project(Project No.17A630016); the Henan provincial government decision tender project(Project No.2016B071); the Henan province Planning Program (Project No.2016G013).

\section{REFERENCES}

[1] Yang Bo. Optimized Study on Solutions for Management Conflict Based on TRIZ [J]. Management review. 2012,03:58-65. (In Chinese)

[2] Lin Yan, Wang Hongqi. The mechanism and strategy of TRIZ theory to promote enterprise innovation [J]. China Science and Technology Forum, 2008,12:57-60+65. (In Chinese)

[3] Jiang Fan, Wang Yijun, Hu YiDan. The mechanism innovation design examples analysis based on TRIZ theory [J]. Journal of Guangzhou University (NATURAL SCIENCE EDITION), 2013,01:75-80. (In Chinese)

[4] Zhu Hong. The change of lifestyle and the evolution of the social function of mobile phone based on the investigation and analysis of middle and low income groups [J]. Journal of Nanjing University (PHILOSOPHY, HUMANITIES AND SOCIAL SCIENCES EDITION), 2011,03:42-50. (In Chinese)

[5] Li Shuchong. IPhone6 prominent mobile phone innovation enterprise [J] industry China Telecom, 2014,10:65. (In Chinese)

[6] Li Suyang, Yang Chunyan, Zhang Xiaowei, et al. The Application of Extenics and the TRIZ Theory in Solving Contradictory Problems [J]. Journal of Guangdong University of Technology, 2013,01:7-12. (In Chinese)

[7] Huang Huiling. Research on Industry Technology Roadmap Based on TRIZ Innovation Theory $[\mathrm{J}]$. Science and Technology Management Research, 2013,13:133-136. (In Chinese)

[8] Wang Xueyuan, Wang Hongqi, Lin Yan. TRIZ Theory's Influence on Enterprise Independent Innovation Performance: Paths and Effectiveness [J]. Journal of information technology, 2013,07:204-207+190. (In Chinese)

[9] Wu Qingyan, Chen Ruisan, Luo Lingling. The advantages and limitations of TRIZ theory [J]. Journal of Liaoning Institute of educational administration, 2013,04:17-21. (In Chinese)

[10] Li Ding, Li Wenjuan. The realization condition and development trend of design concept innovation: a case study of mobile phone design [J]. Journal of Hunan University of Science and Technology (SOCIAL SCIENCE EDITION), 2012,01:144-149. (In Chinese) 